\title{
Demonstration of a Single-Crystal Reflector-Filter for Enhancing Slow Neutron Beams ${ }^{\text {th }}$

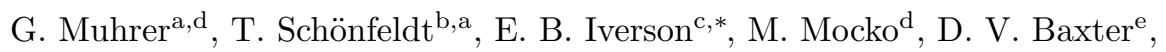 \\ Th. Hügle ${ }^{\mathrm{c}}$, F. X. Gallmeier ${ }^{\mathrm{c}}$, E. B. Klinkby ${ }^{\mathrm{b}, \mathrm{a}}$ \\ ${ }^{a}$ European Spallation Source, Lund, Sweden \\ ${ }^{b}$ Center for Nuclear Technologies, Technical University of Denmark, Roskilde, Denmark \\ ${ }^{c}$ Spallation Neutron Source, Oak Ridge National Laboratory, Oak Ridge, TN USA \\ ${ }^{d}$ Los Alamos Neutron Science Center, Los Alamos National Laboratory, Los Alamos, \\ NM USA \\ ${ }^{e}$ Center for the Exploration of Energy and Matter, Indiana University, Bloomington, \\ IN USA
}

This manuscript has been authored by UT-Battelle, LLC under Contract No. DE-

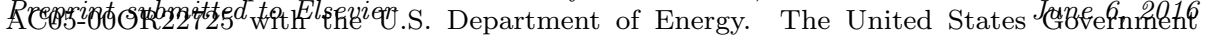
retains and the publisher, by accepting the article for publication, acknowledges that the United States Government retains a non-exclusive, paid-up, irrevocable, world-wide license to publish or reproduce the published form of this manuscript, or allow others to do so, for United States Government purposes. The Department of Energy will provide public access to these results of federally sponsored research in accordance with the DOE Public Access Plan (http://energy.gov/downloads/doe-public-access-plan).

*Corresponding author. Telephone +1 865241 6970; E-mail Address iversoneb@ornl.gov 


\section{Introduction}

In moderators for slow neutron sources, a reflector-filter can be used to enhance the emission of cold neutrons while suppressing the fast neutrons comprising a major source of instrumental background. Some of the neutrons emitted with wavelength less than approximately $4 \AA$ are reflected back into the moderator (reducing the fast neutron emission) and get another chance to be scattered down to desirable energies before being emitted once again. The reflector-filter has only recently been implemented at the Lujan Center, [1] where its observed performance was documented. It increased the source emission above $4 \AA$ by more than a factor of two, while reducing the (for these instruments) undesirable neutron intensity below $1 \AA$ by a factor of four. Unfortunately, the reflectorfilter also suppresses beam intensity between 1 and $4 \AA$ - these neutrons are not necessarily background, but potentially very important. For many scattering instruments this is an acceptable trade-off, but for some scattering instruments, the loss of 1-4 $\AA$ neutrons is unacceptable. It has been theorized that a single crystal can also be used as an effective reflector-filter. [2-4] A single crystal does not have the same sharp Bragg edge associated with a polycrystalline filterthe effective cross section is the same at long wavelengths, but remains low through the 1-4 $\AA$ range as well. We therefore anticipate that a single-crystal reflector-filter will augment the neutron emission at long wavelengths just as the polycrystalline reflector-filter does, but will also enhance (or at least not decrease) the neutron emission at energies $1-4 \AA$ relative to a conventional cold moderator without any reflector-filter.

Reflector-filters, both polycrystalline and single-crystal, were tested on an unreflected pulsed neutron source at the I. V. Kurchatov Institute of Atomic Energy, [3, 4] indicating gains approaching a factor two at long wavelengths from $40 \mathrm{~mm}$ layers of beryllium and quartz, respectively, placed adjacent to the moderator, covering the viewed moderator area as well as significant additional solid angle. As such, the reflector-filters tested there did not distinguish between the gains arising from a (partial) reflector assembly, such as is now common, [5] 
and a reflector-filter blocking only the outgoing beamline within the neutron reflector. Studies in Japan [6] and at the Lujan Center [7, 8] on the beryllium (polycrystalline) reflector-filter reinforce this distinction. The optimum thickness for a polycrystalline beryllium reflector in the unreflected Kurchatov measurement is $20-40 \mathrm{~mm}$, and gives a factor of two low-energy intensity gain. [3] An even thicker beryllium reflector filter (50 mm) was tested in Japan and found to provide no low-energy gain, [6], which those authors attribute to the fact that their test assembly was already fully reflected. At the Lujan Center, the successful deployment of a beryllium reflector-filter in a fully reflected system, which did achieve that factor two gain at low energies, required a much thicker reflector-filter of $120 \mathrm{~mm}$. [7, 8]

This study describes a set of experiments carried out at the Low Energy Neutron Source [9-11] (LENS) between 21 April 2014 and 11 May 2014. The aim of this campaign was to demonstrate the concept of a single-crystal reflectorfilter on a reflected pulsed slow neutron source.

\section{Single Crystal Reflector-Filter Concept}

The concept of a cold beryllium reflector-filter on a reflected pulsed slow neutron source was first proposed in [12], and has later been implemented in the moderator system at the Lujan Center. [1] The concept of a reflector-filter is simple - by placing a thick block of material with high coherent scattering cross section but small incoherent and absorption cross sections on the front face of the moderator (nominally blocking the neutron beam-lines), many fast (energy greater than $1 \mathrm{MeV}$, wavelength less than $3 \times 10^{-4} \AA$ ), slowing-down (energy between $1 \mathrm{eV}$ and $1 \mathrm{MeV}$, or wavelength between $3 \times 10^{-4} \AA$ and $0.3 \AA$ ), and slow (energy below $1 \mathrm{eV}$, wavelength above $0.3 \AA$ ) neutrons which would have leaked out through the neutron beam-ports will have some chance of scattering in the filter, returning to the moderator, and increasing the neutron density in the moderator. Beryllium, with its large but predominantly coherent scattering cross section, is an ideal reflector-filter material. A cold beryllium 
reflector-filter will scatter many neutrons with wavelengths less than that of the so-called Bragg edge at $4 \AA(5 \mathrm{meV})$, but it is very nearly transparent above that wavelength. This property of beryllium is frequently exploited as a beamline filter, [13] taking advantage of the change in the scattering cross section from some 6 barns per atom below $4 \AA$ to less than 0.005 barns per atom above that wavelength (cross sections from ENDF/B-VII.0). As the reflector-filter returns neutrons below $4 \AA$ to the moderator and increases the total neutron density therein, the neutrons have a much higher chance of being emitted at wavelengths above the Bragg edge than would otherwise be the case.

While the cold neutron emission increase at wavelengths above the Bragg edge is significant (as much as a factor two in the right geometry, such as at the Lujan Center, $[1,7,8])$ the major problem with a polycrystalline reflector-filter is the suppression of neutrons with wavelengths below the Bragg edge at $4 \AA$ but still within the slow neutron range useful for neutron scattering. These $1-4 \AA$ neutrons are essential to many instruments, even those primarily considered to be "cold neutron instruments."

The single-crystal reflector-filter [2] exploits the fact that, in a single crystal, only very narrow portions of wavelength-angle phase space meet the Bragg condition and will be scattered by the reflector-filter material. The transmission at long wavelengths (above the Bragg edge) will be just as high as in the polycrystalline case (if not higher, given the possibility of more small-angle scattering in the polycrystal), but the transmission between the Bragg edge and the wavelength at which inter-atomic effects become important (that is, between around 1-4 $\AA$ ) will also be high. This can be exploited in the same way as the conventional reflector-filter to scatter fast neutrons back into the moderator to increase the neutron density, while letting neutrons over the entire wavelength range of interest escape.

As a corollary, a comparison of the wavelength-dependent gain factors of the polycrystalline reflector-filter and the single-crystal reflector-filter (relative to a conventional moderator) should allow us to proportionally attribute the neutron emission gains from that polycrystalline reflector-filter to the wavelength ranges 
below $1 \AA$ and from $1-4 \AA$ :

- If the single-crystal reflector-filter provides a gain factor comparable to the polycrystalline reflector-filter at long wavelengths, and additionally provides a gain $(G(\lambda)>1)$ between $1-4 \AA$, then the reflector-filter benefit is coming primarily from the previously described process acting on fast and slowing-down neutrons $(\lambda<0.5 \AA)$.

- If the single-crystal reflector-filter provides a reduced gain factor at long wavelengths, and imposes a loss $(G(\lambda)<1)$ from $1-4 \AA$ as compared to a conventional moderator, then the reflector-filter benefit is coming primarily from the previously described process acting on slow neutrons below the Bragg edge $(1<\lambda<4) \AA$.

In neutron scattering, it seems that there is no agreement as to whether one should use wavelength or energy for neutrons. In this work, we will use primarily wavelength, for its direct comparison to time-of-flight and easy identification of Bragg edges, even though discussing "fast neutrons," which have energy greater than $1 \mathrm{MeV}$, in terms of wavelength (less than $3 \times 10^{-4} \AA$ ) is unusual.

\section{Experimental setup}

\subsection{LENS Moderator Test Facility}

The Low Energy Neutron Source (LENS) is a small scale neutron facility, producing neutrons from a $13 \mathrm{MeV}$ proton beam impinging on a beryllium target, which we use for moderator research and development. [14] For this experiment LENS was set up to produce $13 \mu$ s pulses at $40 \mathrm{~Hz}$, resulting in a time-averaged beam power of $142 \mathrm{~W}$. The beryllium target is embedded in a cylindrical reflector of $300 \mathrm{~K}$ light water, with a cavity where the moderator system can be lowered from above. The water reflector is surrounded by lead shielding, embedded in alternating layers of lead-epoxy and borax-epoxypolyethylene. The water tank and shielding have an opening enabling four beam-lines to view the moderator location; see Figure 1. When operated as 


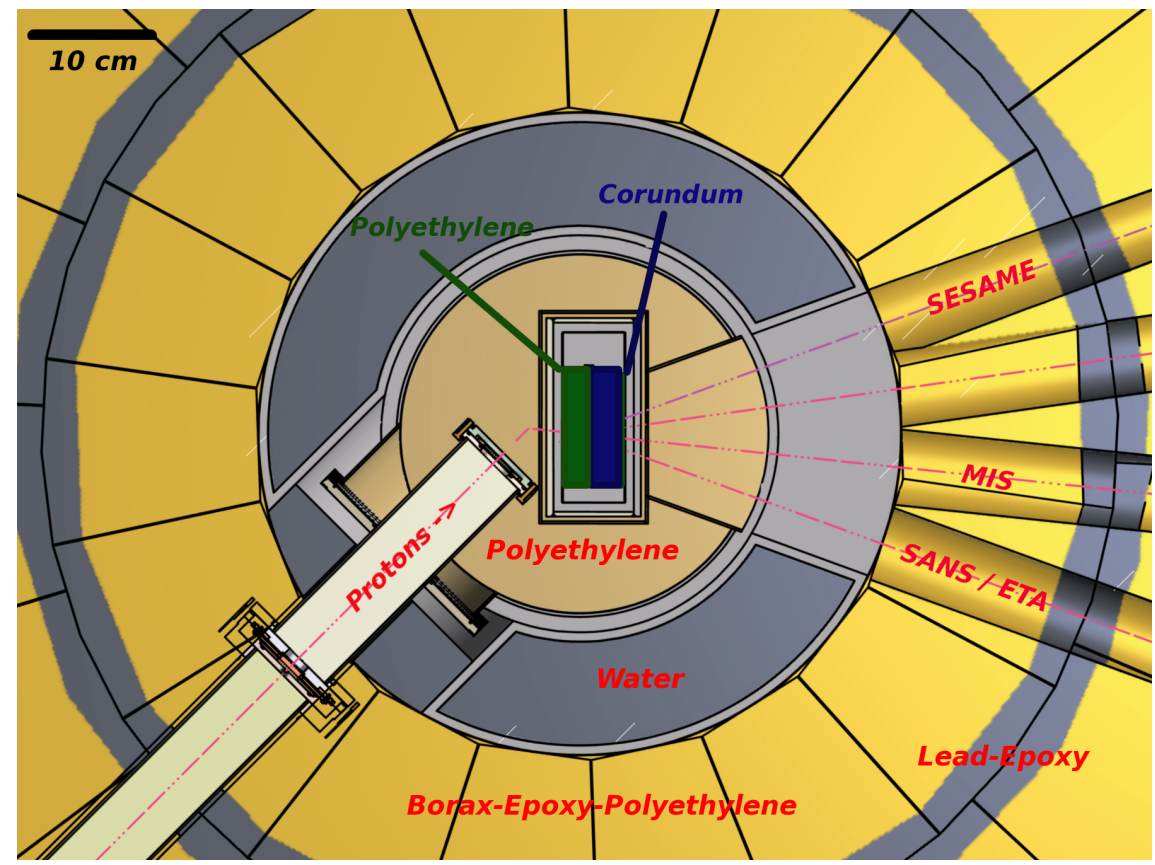

Figure 1: Horizontal cross section through LENS target-moderator-reflector. The green box in the middle represents the polyethylene moderator, while the blue box represents the corundum (or empty) reflector-filter.

a moderator test facility, the moderator usually used in LENS is removed, and test moderator assemblies can be sequentially installed.

\subsection{Instrumentation}

In addition to a proton beam current measuring device (the Q-box) used for normalization, our experimental instrument suite consists of two detectors: a low-efficiency beam monitor within the SANS beam-line and an emission time analyzer we add to the SANS beam-line for moderator tests.

The SANS beam-line beam monitor is a thin low-density ${ }^{3} \mathrm{He}$ detector positioned $(5.25 \pm 0.01) \mathrm{m}$ downstream from the moderator surface along the SANS beam line, which is $20^{\circ}$ away from the moderator surface normal, as shown in Figure 1. This detector provides neutron energy spectra via time-of-flight.

The emission time analyzer is positioned in the SANS sample area during 

in Figure 2. The aluminum rods displace approximately $23 \%$ of the polyethy-

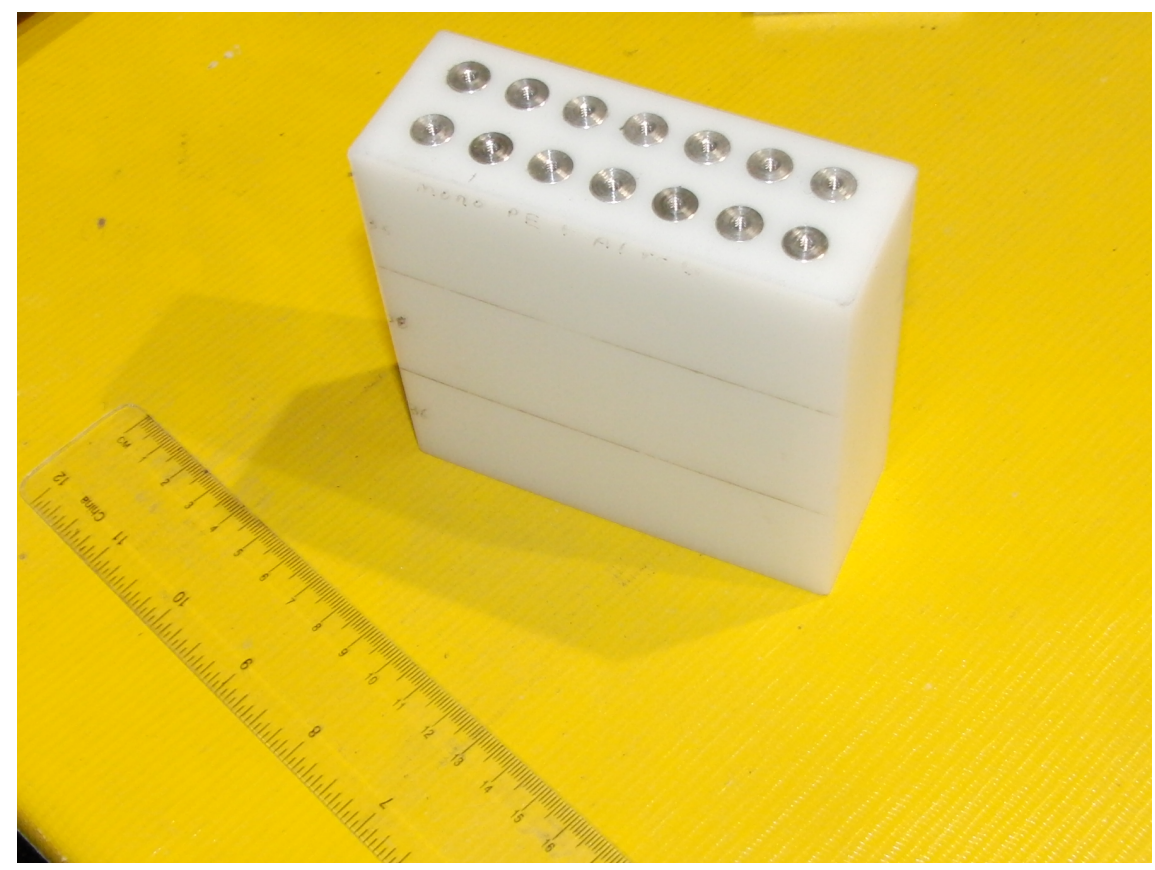

Figure 2: Photograph of polyethylene moderator.

moderator studies. A high mosaicity $10 \mathrm{~K}$ germanium single crystal in the SANS sample position at $8.50 \mathrm{~m}$ diffracts neutrons from the $(n n n)$ series into a scintillator detector positioned in time focused geometry at a nominal Bragg angle of $2 \theta \approx 112^{\circ}$. [15] The emission time analyzer provides the emission time distribution (sometimes called the pulse shape) for several different neutron energies simultaneously.

\subsection{Test Moderator / Reflector-Filter Assemblies}

For these experiments we used a moderator of $25 \mathrm{~mm}$ thick virgin highdensity polyethylene, $110 \mathrm{~mm}$ by $110 \mathrm{~mm}$ on the face, transversely penetrated by $5 \mathrm{~mm}$ diameter thoroughgoing aluminum rods for thermal conductivity, shown
171

lene volume, giving a full-density effective thickness of approximately $19 \mathrm{~mm}$, while significantly flattening the temperature distribution within the moderator at low temperature. The reflector-filter test objects were mounted on the front 
face of the moderator, in identical aluminum vessels, each $51 \mathrm{~mm}$ by $102 \mathrm{~mm}$ by $102 \mathrm{~mm}$. The reflector-filter mounting, on the same cold platform as the moderator itself, is not strictly necessary for the characterization of a reflectorfilter. Indeed, it precludes separate control of the temperature of the moderator and the reflector-filter. However, in these studies we were primarily interested in achieving the lowest practical temperatures in both moderator and reflectorfilter, so no separate control was necessary. The vacuum vessel containing the cryogenic apparatus can be wrapped with absorbing material to decouple the moderator from the long-lived thermalized neutron population within the reflector. For these studies, that decoupler was left off, as our tests were intended to understand the performance of the single-crystal reflector-filter for moderators optimized for long-wavelength intensity, which are typically coupled moderators.

The properties of an ideal material for a single-crystal reflector-filter are similar to those of a conventional reflector-filter: high coherent scattering cross section and low incoherent scattering and absorption cross sections. While beryllium, as used in the Lujan Center reflector-filter, meets these criteria, it is not easily available in large single crystals. Graphite also has a favorable cross section, [2] but is quite expensive in the size and quality needed for our demonstration. We chose to use single crystal corundum $\left(\mathrm{Al}_{2} \mathrm{O}_{3}\right)$ to test the single crystal reflector-filter concept. The test apparatus we used limited the total volume available for a reflector-filter (either single-crystal or polycrystalline) to some $50.8 \mathrm{~mm}$ thickness, well below optimum, but sufficient for this proof of concept. While corundum has a higher capture cross section than desirable, we believe we can correct for this effect.

The single crystal reflector filter was constructed from four single crystal corundum blocks, each $12.7 \mathrm{~mm}$ thick and $102 \mathrm{~mm}$ by $102 \mathrm{~mm}$, which were stacked with the same crystal orientation and positioned at the front face of the polyethylene moderator. As previously stated, the physical constraints near the LENS target preclude a test with a filter of optimal thickness - we describe below how we extrapolate from this geometry-limited experiment to the expected performance of more optimized reflector-filters. The crystallographic orientation 
was confirmed, and the mosaic distribution measured, using the CG1B neutron diffractometer at HFIR [16]. The crystal blocks had mosaic of roughly $0.17^{\circ}$. Each crystal block was oriented with the $c$-axis (as measured by the $\langle 006\rangle$ reflection) emerging within $1^{\circ}$ of the normal to the large face. For each crystal block we identified the orientation of the $\langle\overline{3} 30\rangle$ reflection to be within $4^{\circ}$ of one of the smaller side faces. We named this face the $y$-face. The $\langle 110\rangle$ vector thus emerges nearly normal to the $x$-face, with the $\langle 006\rangle$ vector (the $z$-face) toward the viewer.

We tested four cases - the actual single crystal corundum reflector filter was tested twice. In both configurations using the single-crystal reflector-filter, the crystal lattice was oriented with the $c$-axis emerging along the nominal moderator normal. In the configuration we describe below as $y$-up, the $\langle\overline{3} 30\rangle$ pointed up and the SANS beam-line used for characterization emerges approximately along the $\langle\overline{1} \overline{1} 9\rangle$ direction. In the configuration we describe as $x$-up, the $\langle 110\rangle$ pointed up, and the SANS beam-line emerges approximately along $\langle\overline{1} 110\rangle$. The two remaining cases included one where the crystals were replaced with corundum powder (99.99\% pure with particle size between 0.5 and 1 micron, loaded into an aluminum cell in a helium environment, packed to around $25 \%$ theoretical density), and one where the aluminum casing containing the crystal or powder was left empty (i.e. vacuum).

Each of the four different configurations was first measured at ambient room temperature ( $\sim 300 \mathrm{~K}$ during these experiments) and then at $75 \mathrm{~K}$ without modification to the experimental setup (only the temperature was changed). Only the low-temperature results are described below, as our primary interest for these experiments was in long-wavelength neutron intensity gains. We anticipated possibly significant differences between the $x$-up and $y$-up configurations as the mosaicity of the corundum might result in significant Bragg effects being visible in the transmitted spectra. [17] However, we saw no particular difference between the orientations, and as the $x$-up measurement suffered from poor statistics relative to the $y$-up measurement, we report below only the $y$-up results. 


\section{Analysis}

\subsection{Normalization}

Our data normalization is based on the number of Q-pulses obtained by a measurement of the proton current by a transformer in the proton beam pipe. Each Q-pulse represents $10 \mathrm{nC}$ of proton beam charge passing through the transformer. The recorded data (in counts per time-of-flight bin) from the $1 / v$-SANS beam monitor of known efficiency $\left(4.5 \times 10^{-4}\right.$ counts per neutron at $\left.1 \AA\right)$, when normalized, provide wavelength spectra in neutrons per $\AA$ per nC. The recorded data from the emission time analyzer cannot easily be absolutely normalized, but can easily be normalized to counts per microsecond per $\mathrm{nC}$ enabling relative comparisons. It should be noted that the time in this representation is time-of-flight, not time of exposure, and we use microsecond rather than second to emphasize this distinction. Further details of data analysis can be found elsewhere. [18]

\subsection{Spectral Gain}

The measured spectra are shown in Figure 3. The spectra shown in Figure 3 from the single crystal and powder measurements, when divided by the spectrum from the empty reflector-filter measurement (that is, the spectrum from the bare moderator), produce the gain factors $G_{\mathrm{p}}(\lambda)$ for the polycrystalline powder reflector-filter and $G_{\mathrm{y}}(\lambda)$ for the $y$-up single-crystal reflector-filter shown in Figure 4. Note that the (powder) polycrystalline reflector-filter has approximately one quarter of the neutronic thickness of the single-crystal reflector-filter, due to lower density. If the two samples had the same neutronic thickness, we would expect the suppression of short-wavelength neutron emission to be identical.

The gain factors shown in Figure 4 show the effect of the polycrystalline reflector-filter and the single-crystal reflector-filter. We do observe suppression of short-wavelength neutrons in the beam from the single crystal case, and transmission gradually sets in in the range from $0.3 \AA$ to $1 \AA$ as expected. In the less dense powder sample there is still a suppression of fast neutrons 


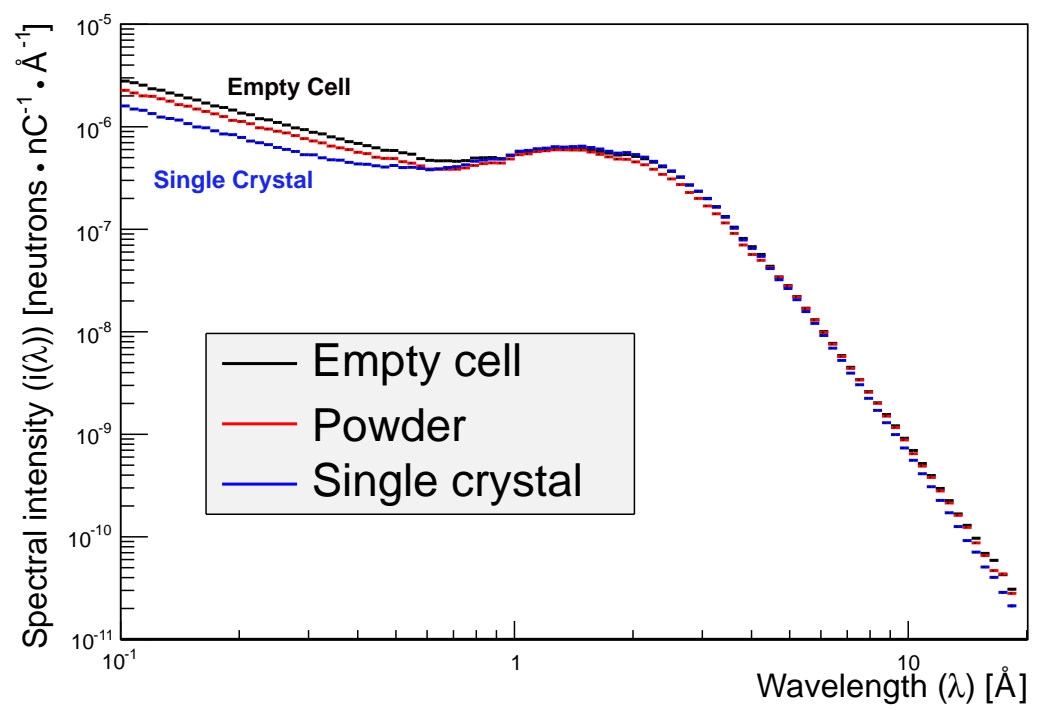

Figure 3: Measured neutron spectra showing suppression of short wavelength neutrons by $75 \mathrm{~K}$ reflector-filters.

and the transmission sets in suddenly at the Bragg edge around $4 \AA$. Another effect, previously observed from polycrystalline reflector-filters, is apparent in the powder case, namely the feature around $1.5 \AA$. [1, 8] This feature appears in both calculated and measured polycrystalline reflector-filter results for the beryllium implementation at the Lujan Center and in our measured results here. We considered whether it might be related to some thermalized component in the reflector-filter itself, but this seems unlikely as the feature appears at too low of a wavelength (around 1.5 A, implying a spectral temperature of around $400 \mathrm{~K}$ from a medium at $70 \mathrm{~K}$ ), and is significantly narrower in wavelength spread than would be expected from a Maxwellian distribution in any event. Similarly, we do not think it related to a thermalized population in the reflector, appearing in the emitted beam after a single scattering within the reflector-filter. With these measurements, we have confirmed that it is not an artifact of either the Lujan Center geometry, or of beryllium itself, and we hope to address its origins in further studies, both experimental and computational. 


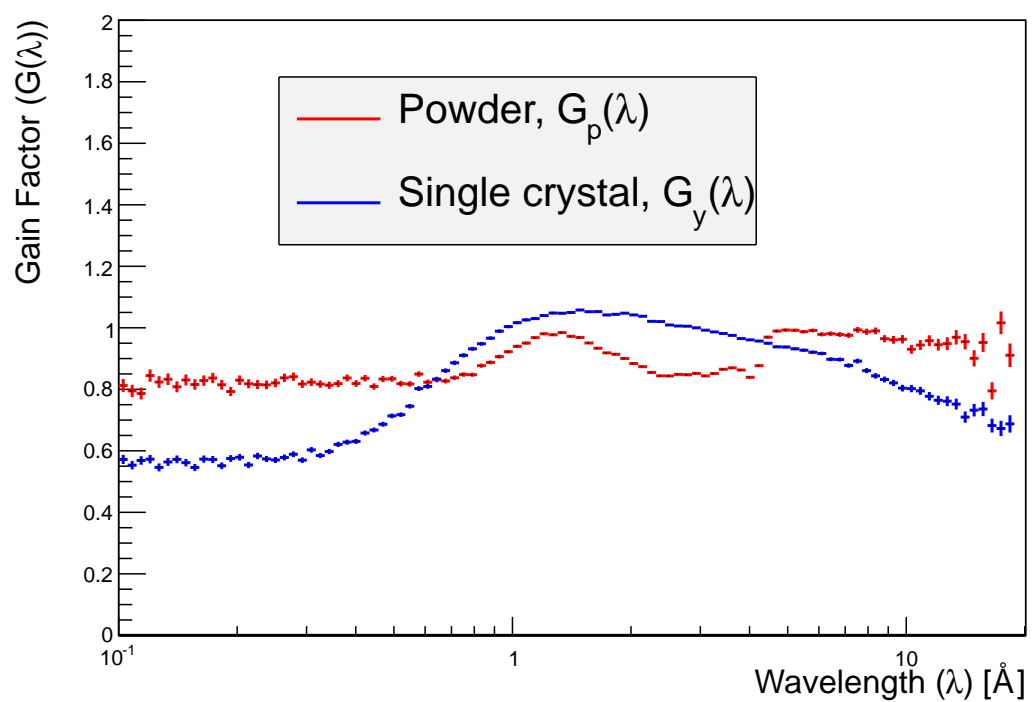

Figure 4: Measured gain factors for the $75 \mathrm{~K}$ polycrystalline and single-crystal reflector-filters studied.

The single-crystal and polycrystalline reflector-filters measured have significantly different neutronic thickness due to a large difference in density (approximately a factor four). To be able to compare the measurements we assume that, were the polycrystalline powder to have the same density as the single crystal, then it would behave identically at wavelengths below some $0.3 \AA$, that is, where structure and orientation effects are negligible. We correct the gain factor of the polycrystalline measurement by treating the gain factor as though it arises from a repeated series of filters, raising it to a power $a$ such that $G_{\mathrm{p}}^{a}\left(\lambda_{0}\right)=G_{\mathrm{y}}\left(\lambda_{0}\right)$ for $\lambda \approx 0.1 \AA$. We apply this correction over the entire wavelength range, assuming that the model of a repeated series of filters is uniformly valid.

By fitting constants to the gain factors at short wavelengths shown in Figure 4, we find $G_{\mathrm{y}}\left(\lambda_{0}\right)=0.566 \pm 0.015$ and $G_{\mathrm{p}}\left(\lambda_{0}\right)=0.818 \pm 0.017$, such that $a=2.83 \pm 0.02$, as applied in Figure 5. Though we believe this to be a good approximation, we would only be able to verify the correction by redoing the experiment using a polycrystalline corundum reflector-filter of equal dimension 


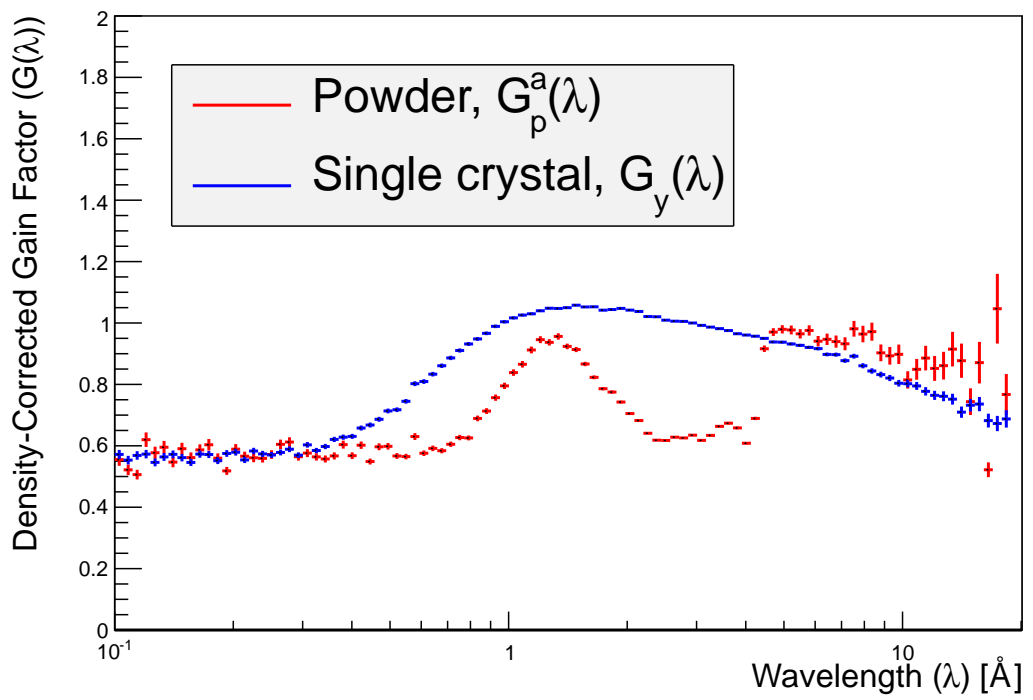

Figure 5: Reflector-filter $(75 \mathrm{~K})$ gain factors corrected for different neutronic thickness.

and density as the single crystal reflector-filter. The apparent gain factor a short wavelength, $G_{\mathrm{y}}\left(\lambda_{0}\right)=0.566 \pm 0.015$, is significantly higher than the transmission of a comparable filter (as opposed to a reflector-filter), for which values from the literature range over $0.15-0.35$. [19-22] The significant variation in the literature is not something we can address at this point, but our apparent gain factor $G_{\mathrm{y}}\left(\lambda_{0}\right)$ is larger yet, indicating that the reflector-filter does indeed boost the intensity within the moderator and at the moderator surface by a significant amount. Strictly speaking, while we can also assume that the reflector-filter provides in-scattering to the beam from the reflector itself, regardless of the intensity within moderator, as well as out-scattering from the beam, we cannot here distinguish among these effects.

It is worth noting that the above comparison also yields an effective estimate of the polycrystalline powder density relative to the single-crystal density; $\rho_{\mathrm{p}}=\rho_{\mathrm{y}} / a$. Using the corundum single-crystal density of $4.03 \mathrm{~g} / \mathrm{cm}^{3}$ we would estimate the effective powder density to be $1.42 \mathrm{~g} / \mathrm{cm}^{3}$, significantly higher than the measured density of $1.06 \mathrm{~g} / \mathrm{cm}^{3}$. That is, the powder appears to have more 
of an effect compared to the thicker single-crystal than its relative density would indicate. We note that the additional scattering power at $0.1 \AA$ could be explained by this additional (unrealistic) $0.36 \mathrm{~g} / \mathrm{cm}^{3}$ of additional corundum, or equally by $0.02 \mathrm{~g} / \mathrm{cm}^{3}$ of water within the corundum powder. Water contamination seems much more plausible. However, that much water would represent an effective $0.1 \mathrm{~cm}$ thickness of water or water ice, which might be expected to have significant effect at long wavelengths.

Figure 5 shows clearly the benefit (increased gain / reduced penalty) of the single-crystal reflector-filter relative to the polycrystalline reflector-filter in the 0.4-4 $\AA$ range, and their similarity at long wavelengths. What Figure 5 also shows is that both reflector-filters are significantly compromised by capture in the corundum (primarily in the aluminum), as can be seen by the fall-off in the gain factor at long wavelengths for both reflector-filters. For a production reflector-filter (whether polycrystalline or single-crystal), one would choose a material with low absorption, such as graphite, diamond, enriched lithium fluoride, beryllium fluoride, enriched lead, or bismuth. To estimate the gain factor of an ideal reflector-filter, polycrystalline or single-crystal, we eliminate the absorption effects by dividing the adjusted gain factors shown in Figure 5 for the single crystal reflector-filter and polycrystalline reflector-filter by the transmission factors (against capture) of a 50.8- mm slab of corundum of density $4.03 \mathrm{~g} / \mathrm{cm}^{3}$ and $1.06 \mathrm{~g} / \mathrm{cm}^{3}$ respectively. To do so, we assume $1 / v$ behavior for both the aluminum and oxygen capture cross sections, with microscopic capture cross sections of $0.233 \mathrm{~b}$ and $0.0002 \mathrm{~b}$, respectively. [23] These observed gain factors $G(\lambda)$, corrected for capture and for full density across $50.8 \mathrm{~mm}$ thickness and defined as $G^{\prime}(\lambda)$, appear in Figure 6 .

Figure 6 clearly shows that low-capture polycrystalline and single crystal reflector-filters would comparable in gain at long wavelengths, and in suppression at short wavelengths, while the single crystal reflector-filter regains the intermediate wavelength neutron emission lost in the polycrystalline reflectorfilter. That is, the single-crystal reflector-filter retains all the long-wavelength advantages of the polycrystalline reflector-filter, without suppressing potentially 


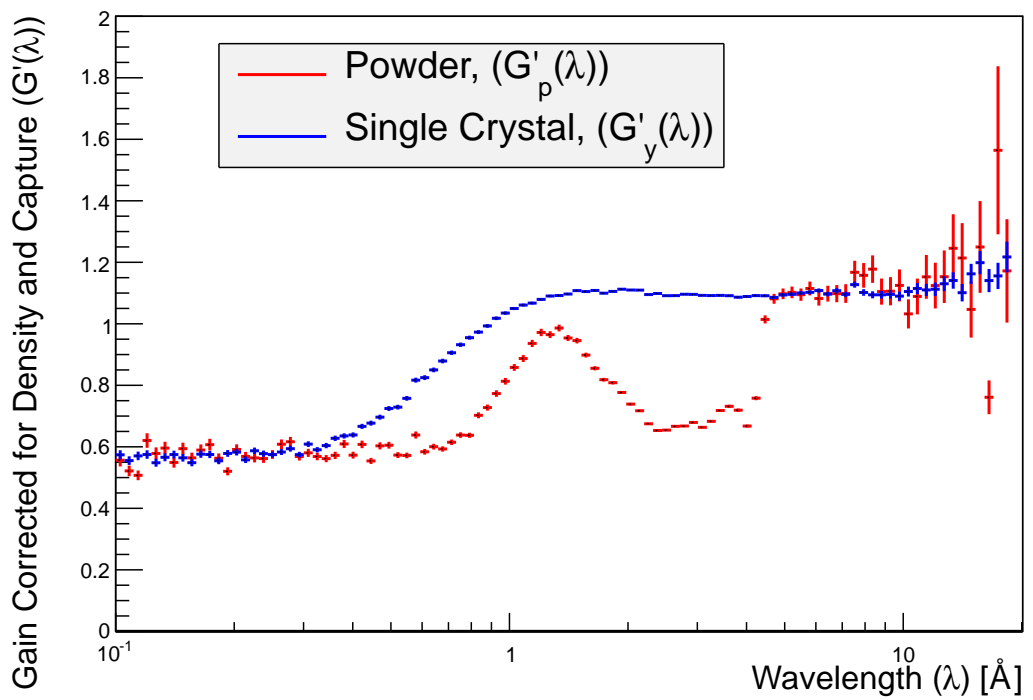

Figure 6: Reflector-filter $(75 \mathrm{~K})$ gain factors corrected for density and capture.

important intermediate wavelength neutrons. Even without the scaling argument applied to $G_{\mathrm{p}}\left(\lambda_{0}\right)$, the fact that $G_{\mathrm{y}}\left(\lambda_{0}\right)$ is larger than a filter transmission at the same wavelength confirms our hypothesis that the gains from a reflectorfilter, whether polycrystalline or single-crystal, accrue from effects on neutrons of wavelength less than $0.1 \AA$ rather than on neutrons of 1-4 $\AA$.

The magnitude of the gains shown in Figure 6 are modest, around $10 \%$ at wavelengths longer than the Bragg edge. The LENS facility is somewhat constrained in the room available for a reflector-filter, precluding prototypical tests. However, the gains shown in Figure 6 indicate that a single-crystal reflector-filter with the same (coherent) scattering cross section as $200 \mathrm{~mm}$ of corundum and negligible capture could provide gains of $50 \%$ at long wavelengths and intermediate wavelengths at the same time, while suppressing background fast neutrons. By extension, a single-crystal reflector-filter with the same scattering power as the beryllium reflector-filter implemented at the Lujan Center could extend the observed factor two gain in neutron intensity for greater than $4 \AA$ to as low as $1 \AA$ wavelength. 


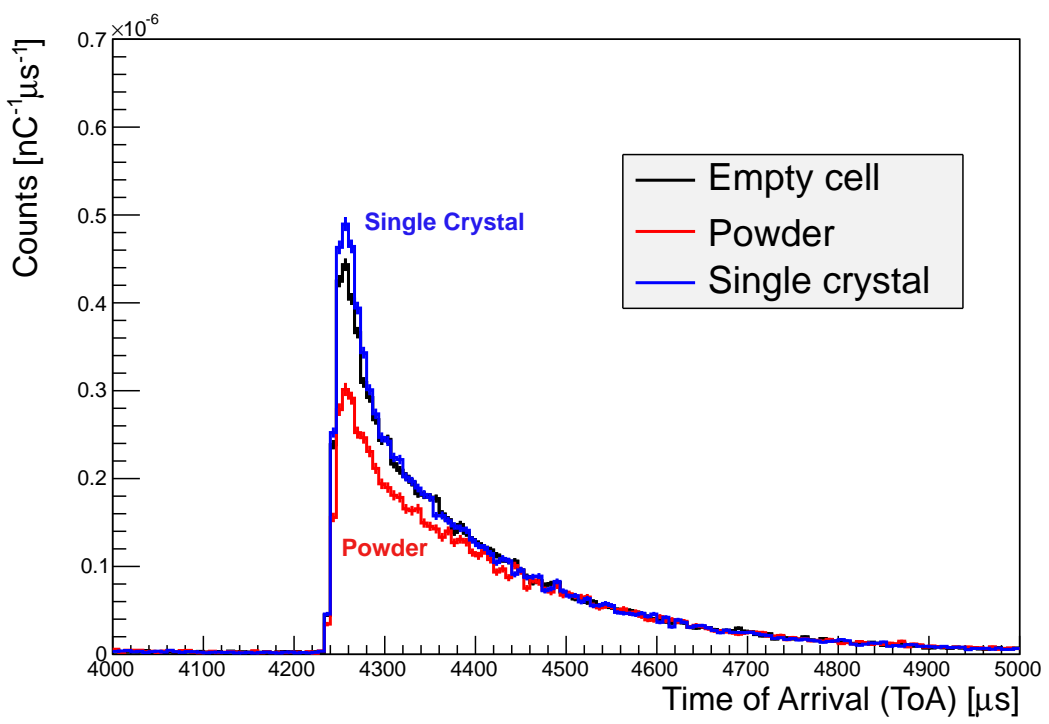

Figure 7: Emission time distributions for $1.81 \AA$ neutrons as measured (without density or capture correction) at $75 \mathrm{~K}$. 
mentioned above are the ratio of the spectra, and approximately corresponds to the ratio of the integrals of these emission time distributions. It is clear from Figure 7 that the intensity change at $1.81 \AA$ occurs primarily in the peak of that distribution. That is, the $5 \%$ gain from the single-crystal reflector-filter as integrated over the emission time distribution is really a $10 \%$ increase in the peak of the distribution. The long-time tails (around $200 \mu$ s after the peak) are unchanged. Similarly, the $10 \%$ loss imposed by the polycrystalline reflector-filter (as compared to the unfiltered system) is really a $30 \%$ loss in peak intensity. Again, the long-time tails are unchanged.

The uncorrected spectral gain at $1.81 \AA, 5 \%$, is not large. But all that gain occurs in the peak of the emission time distribution, and is therefore a gain not only in time-integrated intensity, but also in peak intensity and in available time-of-flight resolution. Instruments for which the only parameter of interest is integrated intensity at a given wavelength will see a modest gain (5\%). Instruments (such as chopper spectrometers) for which the appropriate metric is instantaneous (peak) intensity will see a more significant gain (10\%). Resolution-driven instruments (such as powder diffractometers) with more complicated figures-of-merit which increase with either peak or integrated intensity and also increase with decreasing pulse width will benefit more strongly yet. By looking at the emission time distributions, we can see that the benefit of the single-crystal reflector-filter is even greater than what is indicated solely by the ratios of spectral intensities shown previously. The emission time distributions at $1.35 \AA$ and $1.08 \AA$ show similar effects.

Additionally, the emission time distribution comparison indicates that the action of either reflector-filter does not change the storage time of the moderatorreflector system - further support that the single-crystal reflector-filter gain arises from short-lived epithermal and fast neutron populations being returned to the moderator rather than from a thermal neutron population bouncing back and forth. Finally, we also see that while the polycrystalline reflector-filter does not change the storage time of the moderator-reflector system, it does suppress the peak intensity. From this observation we conclude that the intermediate- 
wavelength neutrons "lost" from the effects of the polycrystalline reflector-filter are not returned to the moderator, and perhaps subsequently emitted at long wavelengths; they are simply lost to the emitted neutron beam.

\section{Conclusion}

We have successfully demonstrated single-crystal and polycrystalline reflectorfilters at LENS. Comparative time-of-flight study reveals that the single-crystal reflector-filter augments intermediate wavelength neutrons as hoped. Our measurements show that the intermediate wavelength neutrons allowed through the single-crystal reflector-filter do not detract from the cold neutron intensity, indicating that the reflector-filter benefit (whether single-crystal or polycrystalline) derives primarily from suppressing fast neutrons of no use to the instruments viewing that moderator.

The reflector-filters we tested in the LENS facility do not provide significant gains for LENS itself. In the first place, corundum, with its high capture cross section, is not an ideal reflector-filter material. In the second place, the LENS geometry, in order to permit easy access to and rapid exchange of test moderators, does not provide sufficient space for an optimized reflector-filter. Our experiments were intended to demonstrate the concept prior to optimizing a single-crystal reflector-filter for a particular application.

\section{Acknowledgements}

Research sponsored by Oak Ridge National Laboratory, managed by UTBattelle, LLC, for the U. S. Department of Energy. Work performed at Oak Ridge National Laboratory is managed by UT-Battelle, LLC, under contract DE-AC05-00OR22725 for the U.S. Department of Energy.

Construction of LENS was supported by the National Science Foundation grants DMR-0220560 and DMR-0320627, the 21st Century Science and Technology fund of Indiana, Indiana University, and the Department of Defense. Operation of LENS is supported by Indiana University, and the experiments 
described in this paper were supported with funds from the US Department of Energy.

This work was supported by Readiness in Technical Base and Facilities (RTBF) which is funded by the Department of Energy's Office of National Nuclear Security Administration. It has benefited from the use of the Manuel Lujan, Jr. Neutron Scattering Center at Los Alamos National Laboratory, which is funded by the Department of Energy's Office of Basic Energy Sciences. Los Alamos National Laboratory is operated by Los Alamos National Security LLC under DOE Contract DE-AC52-06NA25396.

[1] M. Mocko, G. Muhrer, Fourth-generation spallation neutron targetmoderator-reflector-shield assembly at the manuel lujan jr. neutron scattering center, Nuclear Instruments \& Methods In Physics Research Section Aaccelerators Spectrometers Detectors and Associated Equipment 704 (2013) 27-35. doi:10.1016/j.nima.2012.11.103.

[2] G. Muhrer, Cold crystal reflector filter concept (Feb. 2014). arXiv:1402.6314.

URL http://arxiv.org/abs/1402.6314

[3] S. N. Ishmaev, I. P. Sadikov, A. A. Chernyshov, The choice and optimisation of a moderator for a pulsed slow-neutron source, Tech. Rep. IAE 2019, I. V. Kurchatov Institute of Atomic Energy, in Russian (1970).

[4] S. N. Ishmaev, I. P. Sadikov, A. A. Chernyshov, The choice and optimisation of a moderator for a pulsed slow-neutron source; neutron moderation in ice and polythene at low temperatures, Tech. Rep. RL 75129, Rutherford Laboratory, includes English translations of IAE 2019 and IAE 1954 from the I. V. Kurchatov Institute of Atomic Energy. (1975).

[5] J. M. Carpenter, High intensity, pulsed thermal neutron source, US Patent 3,778,627 (Dec. 11 1973).

URL http://www.osti.gov/doepatents/biblio/4326397 
[6] Y. Kiyanagi, Y. Ogawa, N. Kosugi, H. Iwasa, M. Furusaka, N. Watanabe, Further optimization of coupled liquid-hydrogen moderator for intense pulsed neutron source, in: G. S. Bauer, R. Bercher (Eds.), Proceedings of ICANS-XIII, the 13th meeting of the International Collaboration on Advanced Neutron Sources, Vol. II, 1995, p. 654.

URL http://www . neutronresearch. com/proc/?y=1995;n=1; $=0$

[7] G. Muhrer, M. A. Hartl, L. L. Daemen, J. Ryu, Benchmark test of the effectiveness of a nitrogen cooled beryllium reflector-filter, Nuclear Instruments \& Methods In Physics Research Section A-Accelerators Spectrometers Detectors And Associated Equipment 578 (3) (2007) 463-469. doi:10.1016/j.nima.2007.06.004.

[8] G. Muhrer, E. Pitcher, G. Russell, The neutron performance of a pre-moderated beryllium reflector-filter hydrogen moderator system for the Manuel Jr. Lujan Neutron Science Center, Nuclear Instruments and Methods in Physics Research Section A: Accelerators, Spectrometers, Detectors and Associated Equipment 536 (1-2) (2005) 154-164. doi:10.1016/j.nima.2004.07.201.

[9] C. M. Lavelle, D. V. Baxter, A. Bogdanov, V. P. Derenchuk, H. Kaiser, M. B. Leuschner, M. A. Lone, W. Lozowski, H. Nann, B. V. Przewoski, N. Remmes, T. Rinckel, Y. Shin, W. M. Snow, P. E. Sokol, Neutronic design and measured performance of the low energy neutron source (LENS) target moderator reflector assembly, Nuclear Instruments \& Methods In Physics Research Section A-Accelerators Spectrometers Detectors And Associated Equipment 587 (2-3) (2008) 324-341. doi:10.1016/j.nima.2007.12.044.

[10] D. V. Baxter, J. Cameron, M. Leuschner, H. Meyer, H. Nann, W. Snow, LENS-a pulsed neutron source for education and research, Nuclear Instruments and Methods in Physics Research Section A: Accelerators, Spectrometers, Detectors and Associated Equipment 542 (1-3) (2005) 28-31. doi:10.1016/j.nima.2005.01.007. 
[11] D. V. Baxter, J. M. Cameron, V. P. Derenchuk, C. M. Lavelle, M. B. Leuschner, M. A. Lone, H. O. Meyer, T. Rinckel, W. M. Snow, Status of the Low Energy Neutron Source at Indiana University, Nuclear Instruments and Methods in Physics Research Section B: Beam Interactions with Materials and Atoms 241 (1-4) (2005) 209-212. doi:10.1016/j.nimb.2005.07.027.

[12] J. M. Carpenter, R. Kleb, T. A. Postol, R. H. Stefiuk, D. F. R. Mildner, The liquid hydrogen moderator on the ZING-P' pulsed spallation neutron source, Nuclear Instruments and Methods 189 (2-3) (1981) 485-501. doi:10.1016/0029-554X(81)90435-3.

[13] A. D. Taylor, E. J. Wood, J. A. Goldstone, J. Eckert, Lineshape analysis and filter difference method for a high intensity time-of-flight inelastic neutron scattering spectrometer, Nuclear Instruments and Methods in Physics Research 221 (2) (1984) 408-418. doi:10.1016/0167-5087(84)90012-7.

[14] D. V. Baxter, J. Leung, H. Kaiser, S. Ansell, G. Muhrer, E. B. Iverson, P. D. Ferguson, Neutron moderator development research at the Low Energy Neutron Source, Physics Procedia 26 (0) (2012) 117-123. doi:10.1016/j.phpro.2012.03.016.

[15] K. F. Graham, J. M. Carpenter, Pulsed moderator studies using a time focussed crystal spectrometer, Nuclear Instruments and Methods 85 (2) (1970) 163-171. doi:10.1016/0029-554X(70)90236-3.

[16] L. Crow, L. Robertson, H. Bilheux, M. Fleenor, E. B. Iverson, X. Tong, D. Stoica, W. T. Lee, The CG1 instrument development test station at the High Flux Isotope Reactor, Nuclear Instruments and Methods in Physics Research Section A: Accelerators, Spectrometers, Detectors and Associated Equipment 634 (1, Supplement) (2011) S71-S74. doi:10.1016/j.nima.2010.06.213.

[17] A. Freund, H. Friedrich, W. Nistler, R. Scherm, Neutron transmission properties of perfect silicon crystals, Nuclear Instruments and Methods 
in Physics Research Section A: Accelerators, Spectrometers, Detectors and Associated Equipment 234 (1) (1985) 116-121. doi:10.1016/01689002(85)90815-0.

[18] E. B. Iverson, D. V. Baxter, G. Muhrer, S. Ansell, R. Dalgliesh, F. X. Gallmeier, H. Kaiser, W. Lu, Enhancing neutron beam production with a convoluted moderator, Nuclear Instruments and Methods in Physics Research Section A: Accelerators, Spectrometers, Detectors and Associated Equipment 762 (0) (2014) 31-41. doi:10.1016/j.nima.2014.04.047.

[19] D. C. Tennant, Performance of a cooled sapphire and beryllium assembly for filtering of thermal neutrons, Review of Scientific Instruments 59 (2) (1988) 380-381. doi:10.1063/1.1140212.

[20] D. F. R. Mildner, M. Arif, C. A. Stone, R. K. Crawford, The neutron transmission of single-crystal sapphire filters, Journal of Applied Crystallography 26 (3) (1993) 438-447. doi:10.1107/s0021889893000433.

[21] D. F. R. Mildner, G. P. Lamaze, Neutron transmission of singlecrystal sapphire, Journal of Applied Crystallography 31 (1998) 835-840. doi:10.1107/S0021889898005846.

[22] F. Cantargi, J. R. Granada, R. E. Mayer, Thermal neutron scattering kernels for sapphire and silicon single crystals, Annals of Nuclear Energy 80 (2015) 43-46. doi:10.1016/j.anucene.2015.01.020.

[23] V. F. Sears, Neutron scattering lengths and cross sections, Neutron News 3 (3) (1992) 26-37. doi:10.1080/10448639208218770. 